$\Sigma^{-}$. Qualitatively there is very little difference between the $\Lambda$ and $\Sigma$ spectra. At higher energies the spectra peak more sharply.

Figure 5 presents the total cross sections for $\Lambda$ and $\Sigma^{-}$. The main qualitative difference, typical of $V-A$ versus $V+A$ is the much more rapid rise for $\Lambda$ production. This is a possible way to verify that in the $\Sigma^{-}$ interaction $V / A$ is positive.
It is also of some interest to use our asymptotic formulas to make estimates of the hyperon to nucleon ratios. If we use Eq. (32) and choose $a^{2} / 2=M_{\rho}^{2}$ or $M_{K^{*}}$, then the results are

and

$$
\sigma_{\Lambda} / \sigma_{N} \rightarrow 0.078 \approx 1 / 13
$$

$$
\sigma_{\Sigma} / \sigma_{N} \rightarrow 0.055 \approx 1 / 18
$$

\title{
Rates of Nuclear Reactions in Solid-Like Stars*
}

\author{
RICHARD A. WOLF $\dagger$ \\ California Institute of Technology, Pasadena, California
}

(Received 9 November 1964)

\begin{abstract}
In stellar matter as cool and dense as the interior of a white dwarf, the Coulomb energies between neighboring nuclei are large compared to the kinetic energies of the nuclei. Each nucleus is constrained to vibrate about an equilibrium position, and the motion of the nuclei in the interior of a white dwarf is similar to the motion of the atoms in a solid or liquid. We propose a solid-state method for calculating the rate at which a nuclear reaction proceeds between two identical nuclei oscillating about adjacent lattice sites. An effective potential $U(\mathbf{r})$ derived by analyzing small lattice vibrations is used to represent the influence of the Coulomb fields of the lattice on the motion of the two reacting nuclei. The wave function describing the relative motion of the two reacting particles is obtained by solving the Schrödinger equation containing the effective potential $U(\mathbf{r})$. From this wave function, we derive an expression for the reaction rate. The rates of the $p+p$ and $\mathrm{C}^{12}+\mathrm{C}^{12}$ reactions calculated using this solid-state method are typically 1 to 10 orders of magnitude smaller than those calculated by the method previously suggested by Cameron.
\end{abstract}

\section{INTRODUCTION}

$T$ HE motions of nuclei in the interiors of cool, dense stars resemble the motions of atoms in solids or liquids. The mean free path between collisions suffered by a given nucleus is much smaller than the average distance between nuclei and may be comparable to the particle's quantum-mechanical wavelength. Each nucleus is therefore forced to oscillate about a fixed position in a lattice structure. ${ }^{1}$

Reactions between charged particles in stars are inhibited by the small probability of penetrating the Coulomb barrier between nuclei. However, the probability of penetrating the barrier increases rapidly with the energies of the colliding particles. In most stars, the effective energies are due primarily to thermal motions. In stars as cold as white dwarfs, the thermal energies alone are too small to allow charged particles to react at significant rates. However, the Coulomb potential of the lattice combined with the ground-state vibrational energy of the reacting nuclei can, at high densities, enable nuclei at adjacent lattice sites to react rapidly even at zero temperature.

* Supported in part by the U. S. Office of Naval Research [Nonr-220(47)] and the National Aeronautics and Space Administration [NGR-05-002-028].

$\dagger$ National Science Foundation Predoctoral Fellow in Physics.

${ }^{1}$ E. E. Salpeter, Astrophys. J. 134, 669 (1961).
It is important that one be able to calculate the rates of reactions occurring at high densities and low temperatures, reactions to which Cameron ${ }^{2}$ has applied the name "pycnonuclear." Cameron has suggested that such reactions might be the source of energy for nova explosions. A knowledge of the rates of pycnonuclear reactions would also be useful in mathematical studies of white dwarfs. From the rates of reactions at high densities, one can infer certain limitations on the possible compositions of the interiors and envelopes of whitedwarf stars, compositions which would otherwise be completely unknown. ${ }^{3}$ Any future attempts to evolve stellar models into the white-dwarf state from higher temperature configurations will also require detailed knowledge of pycnonuclear reaction rates.

In this paper we develop a method for finding the rate at which nuclear reactions proceed between particles vibrating about adjacent lattice sites. For reactions between particles with $Z \geq 2$, the solid-state approach applies to the temperatures and densities in region I of Fig. 1. Figure 1 also shows typical central temperatures and densities for various types of stars.

We consider primarily reactions in a lattice of identical nuclei, although we do suggest a rough model for

${ }^{2}$ A. G. W. Cameron, Astrophys. J. 130, 916 (1959).

${ }^{3}$ T. Hamada and E. E. Salpeter, Astrophys. J. 134, 683 (1961). 
generalizing the method to include reactions in lattices with arbitrary compositions. A more accurate treatment of reactions in dense stars with complicated compositions would require detailed analysis of the structures of lattices containing more than one nuclear species.

At the high densities of interest here, the motions of any pair of nuclei are strongly coupled to the motions of other nuclei nearby. In order to compute the mean lifetime for a reaction between two adjacent nuclei without solving the complete many-body problem exactly, we make the fundamental assumption that the effect of the rest of the lattice on the relative motion of the two reacting particles can be adequately represented by a static potential $U(\mathbf{r})$. The reaction rate depends strongly on $U(\mathbf{r})$ through the barrier-penetration factor. In Sec. II, we analyze the small vibrations of the lattice to find $U(\mathbf{r})$. Then in Sec. III, we solve the Schrödinger equation for the wave function characterizing the relative motion of the two reacting particles. Having found this wave function, we derive an expression for the reaction rate. Section IV contains a discussion of the limitations of the solid-state treatment. We also consider in Sec. IV the problem of generalizing the method to include reactions between nonidentical nuclei. In Sec. V, we present numerical results for the rates of the $p+p$ and $\mathrm{C}^{12}+\mathrm{C}^{12}$ reactions. Our method predicts rates several orders of magnitude slower than those obtained using the procedure suggested by Cameron. ${ }^{2}$ Salpeter ${ }^{4}$ has developed a way of calculating reaction rates at temperatures higher than those covered by the solidstate method; our results are consistent with those of Salpeter.

\section{ESTIMATION OF THE EFFECTIVE POTENTIAL}

\section{A. General Discussion}

The strong Coulomb forces between nuclei in a lattice greatly complicate the calculation of reaction rates at high densities. Each nucleus experiences Coulomb forces due to many neighboring particles. To compute the reaction rate per unit volume exactly, one would have to solve the complete many-body problem including all the nuclei in the lattice. This many-body problem seems tractable only for the case of small displacements of the nuclei from positions in a periodic lattice, the case to which the phonon approach of solidstate physics is applicable.

We cannot calculate reaction rates, however, by relying just on the phonon theory to describe the motion of nuclei under the influence of lattice Coulomb fields. A nuclear reaction between two particles must involve their approaching one another to within a distance of the order of the nuclear radius, which is much smaller than $b_{\mathrm{nn}}$, the nearest-neighbor distance. The phonon theory does not apply to such large displacements from equilibrium. We do know, however, that for small

${ }^{4}$ E. E. Salpeter, Australian J. Phys. 7, 373 (1954).

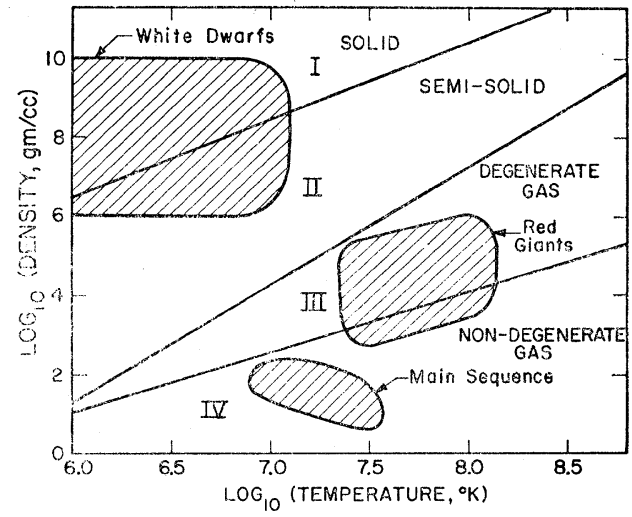

FIG. 1. Central temperatures and densities of various types of stars. The solid-state approach to nuclear reactions applies to region I on the figure. In region II, most nuclear motion is vibrational, but the nuclei most likely to react have enough energy to break through the lattice. In regions III and IV, the nuclei move like atoms in a gas. In region III, the electrons are degenerate, while in region IV they are nondegenerate.

separations between nuclei, the relative motion of the two nuclei is influenced primarily by a potential $Z^{2} e^{2} r^{-1}$, and the forces due to the rest of the lattice are not important.

We assume that the relative motion of two nuclei oscillating about adjacent lattice sites can be adequately represented by motion in some potential $V(\mathbf{r})$. We require that $V(\mathbf{r}) \rightarrow Z^{2} e^{2} r^{-1}$ as $r \rightarrow 0$ and use the results of the phonon analysis to determine $V(\mathbf{r})$ for small displacements from equilibrium, i.e., for $\mathbf{r}$ approximately equal to the vector between the equilibrium positions of the two nuclei. In this way, one can reduce the manybody problem involving all the nuclei in the lattice to one involving just the relative motion of two particles. By proper choice of the potential, we can accurately approximate the effects of motions of the neighboring nuclei.

In this section we treat only identical nuclei, each having mass $M$ and charge $Z e$. We consider the rate at which a nuclear reaction proceeds between two of these nuclei, labeled 1 and 2 . Let the relative displacement of the two nuclei be given by $\mathbf{r}=\mathbf{r}_{1}-\mathbf{r}_{2}$, and let the components of $\mathbf{r}$ be $x, y$, and $z$. The mass characterizing the relative motion is given by

$$
\mu=\frac{1}{2} M \text {. }
$$

Let the equilibrium positions of the particles be separated by a distance $b_{\mathrm{nn}}$ along the $z$ axis, where $b_{\mathrm{nn}}$ is the nearest-neighbor distance characteristic of the lattice. The potential $V(\mathbf{r})$ acting on the relative motion of the two neighboring nuclei must have a minimum at $\left(0,0, b_{\mathrm{nn}}\right)$. Near the minimum point, the potential has the form

$V(\mathbf{r}) \approx V\left(0,0, b_{\mathrm{nn}}\right)+\frac{1}{2} \mu\left[\Omega_{x}{ }^{2}\left(x^{2}+y^{2}\right)+\Omega_{z}{ }^{2}\left(z-b_{\mathrm{nn}}\right)^{2}\right]$, (II.2)

if the lattice is, as expected, symmetric under the operations $(x \rightarrow-x, y \rightarrow y),(x \rightarrow x, y \rightarrow-y)$, and 
B 1636

$(x \rightarrow y, y \rightarrow-x)$. In Sec. IIB we use the phonon analysis of lattice vibrations to determine the values of $\Omega_{x}$ and $\Omega_{z}$.

We note that the total effective potential can be separated into two parts, one representing the static Coulomb field between nuclei 1 and 2, and the other representing the effective potential due to the other nuclei in the lattice. That is, we can write

$$
V(\mathbf{r})=Z^{2} e^{2} r^{-1}+U(\mathbf{r}) .
$$

Since nuclei 1 and 2 are assumed identical, the potential $U(\mathbf{r})$ satisfies the relation

$$
U(\mathbf{r})=U\left(\mathbf{r}_{1}-\mathbf{r}_{2}\right)=U\left(\mathbf{r}_{2}-\mathbf{r}_{1}\right)=U(-\mathbf{r}),
$$

which implies that

$$
\nabla U(0,0,0)=0 .
$$

We define the zero of energy by the relation

$$
U(0,0,0)=0 .
$$

Equations (II.2)-(II.5) express all our knowledge of $U(\mathbf{r})$. They determine the value and gradient of $U(\mathbf{r})$ at the origin and the gradient and second derivatives of $U(\mathbf{r})$ at $\left(0,0, b_{\mathrm{nn}}\right)$. Equations (II.2)-(II.5) obviously do not determine $U(\mathbf{r})$ uniquely for all $\mathbf{r}$.

We must now consider the effects of our incomplete knowledge of $U(\mathbf{r})$ on the calculated reaction rate. It can be shown that the potential $U(\mathbf{r})$ affects the reaction rate mainly through a barrier penetration factor $P(E)$, where

$$
P(E)=\exp \left[-(8 \mu)^{1 / 2} \hbar^{-1} I_{p}\right] .
$$

The factor $I_{p}$ in Eq. (II.6a) is defined by

$$
I_{p}=\int_{R}^{r_{c}}\left[Z^{2} e^{2} r^{-1}+U(0,0, r)-E\right]^{1 / 2} d r,
$$

where $E$ is the energy of the relative motion, $R$ is the nuclear radius, and $r_{c}$ is the classical turning point radius defined by

$$
E-U\left(0,0, r_{c}\right)=Z^{2} e^{2} r_{c}^{-1} .
$$

For $r$ near $r_{c}$, the quantity $U(0,0, r)-E$ makes an important contribution to the integrand in Eq. (II.6b). Fortunately, for $r$ near $r_{c}$, the quantity $U(0,0, r)-E$ can be determined accurately from Eqs. (II.2) and (II.3). For $r$ near $b_{\mathrm{nn}}$, the potential $V(\mathbf{r})$ is accurately described by Eq. (II.2). Since we assume that the vibrations are small, i.e.,

$$
\left(b_{\mathrm{nn}}-r_{c}\right) b_{\mathrm{nn}}{ }^{-1} \ll 1,
$$

the harmonic oscillator approximation of Eq. (II.2) is accurate in the region where the wave function is large. Thus, the eigenstates of the Schrödinger equation with potential $V(\mathbf{r})$ can be labeled by harmonic oscillator quantum numbers $n_{x}, n_{y}$, and $n_{z}$, and the relation

$$
\begin{aligned}
E=E\left(n_{x}, n_{y}, n_{z}\right)=\left(n_{x}+n_{y}+1\right) \hbar \Omega_{x} & \\
& +\left(n_{z}+\frac{1}{2}\right) \hbar \Omega_{z}+V\left(0,0, b_{\mathrm{nn}}\right)
\end{aligned}
$$

gives the energy eigenvalue for the state $\left(n_{x}, n_{y}, n_{z}\right)$.

Equation (II.8) accurately establishes $E-V\left(0,0, b_{\mathrm{nn}}\right)$ for any given state, while Eqs. (II.2) and (II.3) accurately determine $U(0,0, r)-V\left(0,0, b_{\mathrm{nn}}\right)$ for $r$ near $r_{c}$. Hence the quantity $U(0,0, r)-E$ is known for $r$ near $r_{c}$.

However, Eqs. (II.2)-(II.5) do not accurately determine $U(0,0, r)-E$ for $r \ll r_{c}$. Fortunately the integral in Eq. (II.6b) does not depend strongly on $U(0,0, r)-E$ for small $r$, since

$$
Z^{2} e^{2} r^{-1} \gg|U(0,0, r)-E|,
$$

if $r \ll r_{c}$. In order to minimize the error in the barrier penetration integral $I_{p}$ due to our incomplete knowledge of $U(0,0, r)-E$, we assume $U(\mathbf{r})$ can be represented in the simple form

$$
U(\mathbf{r})=k_{2} r^{2}+k_{3} r^{3}+k^{\prime}\left(x^{2}+y^{2}\right) .
$$

Substituting Eq. (II.9a) in Eq. (II.3) and comparing the result to Eq. (II.2) for $r$ near $\left(0,0, b_{\mathrm{nn}}\right)$ yields

$$
\begin{gathered}
k_{2}=2 Z^{2} e^{2} b_{\mathrm{nn}}{ }^{-3}-\frac{1}{2} \mu \Omega_{z}{ }^{2}, \\
k_{3}=-Z^{2} e^{2} b_{\mathrm{nn}}{ }^{-4}+\frac{1}{3} \mu \Omega_{z}{ }^{2} b_{\mathrm{nn}}{ }^{-1}, \\
k^{\prime}=\frac{1}{2} \mu \Omega_{x}{ }^{2} .
\end{gathered}
$$

(The oscillator frequencies $\Omega_{x}$ and $\Omega_{z}$ will be determined in Sec. IIB.) We have assumed that $U(\mathbf{r})$ takes the simplest form consistent with Eqs. (II.2)-(II.5). Further investigation has shown that several other smooth forms assumed for $U(\mathbf{r})$, forms which are also consistent with Eqs. (II.2)-(II.5), yield values of $I_{p}$ within a few percent of that given by the $U(\mathbf{r})$ of Eq. (II.9a).

\section{B. Lattice Dynamics}

\section{General Discussion}

In this subsection we use a normal-mode analysis to show that the relative motion of particles 1 and 2 can, for small displacements, be represented by motion in a harmonic-oscillator potential. We then compute the frequencies $\Omega_{x}$ and $\Omega_{z}$ characterizing the oscillator potential.

The electrons are highly degenerate at the temperatures and densities to which the solid-state method applies. The energy of the Coulomb interaction between an electron and a nucleus is comparable to the average electron kinetic energy only at distances small compared to the electron's wavelength. Consequently, the fields of individual nuclei cannot significantly affect the electron wave functions. The electrons can react only to lattice vibrations with very long wavelengths. By solving the Thomas-Fermi equation for the electron 
distribution, one can show ${ }^{1}$ that the electron motion affects only a negligible part of the vibrational spectrum as long as

$$
b_{\mathrm{nn}} \ll a_{0} Z^{-1 / 3},
$$

where $a_{0}$ is the Bohr radius. Since inequality (II.10) always holds under the conditions to which the solidstate model applies, we assume a uniform distribution of electrons.

The total potential of the system of electrons and nuclei is then the sum of the following three terms: (1) the electron-electron potential energy, which does not depend significantly on the positions of the nuclei; (2) the potential energy of interaction between the uniform distribution of electrons and the lattice of nuclei; and (3) the energy of the Coulomb interactions among the nuclei themselves.

For small displacements of the nuclei from their equilibrium positions, the potential energy can be written to good accuracy in the form

$$
W=W_{0}+W_{2} \text {, }
$$

where $W_{0}$ is independent of the nuclear displacements, and $W_{2}$ is a homogeneous polynomial of second order in the displacements. Using the usual normal mode procedure, ${ }^{5}$ we can find linear combinations $Q_{s}$ of the displacements of the nuclei such that the total Hamiltonian of the system of nuclei can be written in the form

$$
H=\frac{1}{2} \sum_{s}\left(P_{s}^{2} M_{s}^{-1}+M_{s} \omega_{s}^{2} Q_{s}^{2}\right),
$$

where, classically,

$$
P_{s}=M_{s} \dot{Q}_{s},
$$

and $M_{s}$ and $\omega_{s}{ }^{2}$ are constants independent of the nuclear displacements. Quantizing the system, we find that the wave function describing the nuclear displacements is the product of the harmonic oscillator wave functions for all the normal mode oscillators.

We shall find in Sec. IV that the solid-state approach applies primarily to temperatures such that $k T \hbar^{-1}$ is small compared to most of the normal mode frequencies. It is therefore reasonable to consider the zero-temperature limit and assume that all of the normal mode oscillators are in their ground states.

The ground-state harmonic oscillator wave function is a simple Gaussian, and the product of the groundstate wave functions of all the normal mode oscillators can be written

$$
\Psi=A \exp \left[-\sum_{s} \frac{1}{2}\left(M_{s} \omega_{s} \hbar^{-1}\right) Q_{s}{ }^{2}\right],
$$

where $A$ is a normalization constant. Since the $Q_{s}$ are linear combinations of the displacements, the exponent in Eq. (II.14) could also be written as a homogeneous polynomial of second order in the displacements. We are interested only in the relative motion of particles 1

${ }^{5}$ For a discussion of the normal mode approach as applied to solid lattices, see, for example, J. M. Ziman, Electrons and Phonons (Oxford University Press, London, 1960), Chap. 1. and 2. To find the probability distribution for the relative displacement of particles 1 and 2, we integrate $|\Psi|^{2}$ over the displacements of all the nuclei except 1 and 2 , and then integrate over the displacement of the center of mass of particles 1 and 2. The successive integrations of $|\Psi|^{2}$ over the displacements do not alter the general functional form. Each integration yields a pure exponential with a homogeneous polynomial of second order as the exponent. Assuming the lattice invariant under the operations $(x \rightarrow-x, y \rightarrow y)$, $(x \rightarrow x, y \rightarrow-y)$, and $(x \rightarrow y, y \rightarrow-x)$, we obtain an expression of the form

$P(\mathbf{r})=A^{\prime} \exp \left\{-\mu \hbar^{-1}\left[\Omega_{x}\left(x^{2}+y^{2}\right)+\Omega_{z}\left(z-b_{\mathrm{nn}}\right)^{2}\right]\right\}$

for the probability distribution of the relative positions of particles 1 and 2 .

The probability distribution described by Eq. (II.15) is identical with that of a three-dimensional harmonic oscillator in its ground state. Despite the complicated effects of lattice vibrations on the relative motion of the two adjacent nuclei, the probability distribution for small displacements in the relative positions of the two nuclei is the same as it would be if the relative motion of the two nuclei were subjected to a static harmonic oscillator potential. Thus, we have only to find the proper oscillator frequencies $\Omega_{x}$ and $\Omega_{z}$.

\section{Finding the Oscillator Frequencies}

We know that for small displacements, the probability amplitude is a three-dimensional Gaussian. The remaining problem is to find the widths of the Gaussian in the transverse and longitudinal directions. The widths are related to the oscillator frequencies by

$$
\left\langle x^{2}\right\rangle_{0}=\int d x \int d y \int d z x^{2} P(\mathbf{r})=\hbar\left(2 \mu \Omega_{x}\right)^{-1},
$$

and

$$
\begin{aligned}
& \left\langle\left(z-b_{\mathrm{nn}}\right)^{2}\right\rangle_{0}=\int d x \int d y \int d z\left(z-b_{\mathrm{nn}}\right)^{2} P(\mathbf{r}) \\
& \text { if } \\
& =h\left(2 \mu \Omega_{z}\right)^{-1} \text {, } \\
& \int d x \int d y \int d z P(\mathbf{r})=1 \text {. }
\end{aligned}
$$

The phonon approach of solid-state physics provides an easy way of calculating $\left\langle x^{2}\right\rangle_{0}$ and $\left\langle\left(z-b_{\mathrm{nn}}\right)^{2}\right\rangle_{0}$. For the case of a periodic lattice, the normal mode vibrations can be described as lattice waves with given wave numbers and polarizations. The characteristic frequencies and the polarization vectors were calculated numerically for several thousand wave numbers in the first Brillouin zone, ${ }^{6}$ and the expectation values $\left\langle x^{2}\right\rangle_{0}$ and $\left\langle\left(z-b_{n n}\right)^{2}\right\rangle_{0}$ were computed using an average over the first Brillouin zone. These expectation values, when substituted in Eqs. (II.16a) and (II.16b), yield the

\footnotetext{
${ }^{6}$ Normal mode frequencies in a lattice of like charges have also been computed by W. J. Carr, Jr., Phys. Rev. 122, 1437 (1961).
} 
B 1638

following results :

and $\Omega_{x}=1.28 \omega_{0}$,

where

$$
\Omega_{z}=1.88 \omega_{0}
$$

$$
\omega_{0}=Z e\left(M b^{3}\right)^{-1 / 2} \text {, }
$$

and $b^{-3}$ is the number density of nuclei in the lattice. These numerical values are expected to be accurate to within $1 \%$ for the physical model adopted here. By substituting Eqs. (II.17a) and (II.17b) in Eqs. (II.9b)(II.9d) one can find the parameters $k_{2}, k_{3}$, and $k^{\prime}$ in the expression for $U(\mathbf{r})$.

We have used the normal mode analysis of lattice vibrations to determine the parameters $\Omega_{x}$ and $\Omega_{z}$ characterizing the effective potential $V(\mathbf{r})$ acting on the relative motion of the two reacting particles. In Sec. III, we solve the Schrödinger equation containing $V(\mathbf{r})$ for the wave function of the relative motion of the reacting particles. Before proceeding to solve the Schrödinger equation, however, we should consider two related problems.

\section{Nonzero Temperature}

We have treated only the case where all the oscillators are in their ground states. For most of the temperatures to which the solid-state model applies, nearly all of the oscillators are in fact in their ground states. However, we can calculate the average expectation values of $x^{2}$ and $\left(z-b_{\mathrm{nn}}\right)^{2}$ for any given temperature using the same phonon approach. For all temperatures, these average expectation values are within about $20 \%$ of those obtained using the simple harmonic oscillator model with the frequencies $\Omega_{x}$ and $\Omega_{z}$ given by Eqs. (II.17a) and (II.17b). Thus, we expect that the approximate potential well of Eq. (II.2) describes the relative motion even for nonzero temperatures.

\section{Comparison with the Static Model}

We have determined the lattice potential $U(\mathbf{r})$ by examining small vibrations of the lattice. The strong coupling between the relative motion of two reacting particles and the motion of neighboring nuclei is thus taken into account approximately.

The frequencies $\Omega_{x}$ and $\Omega_{z}$ can be obtained more easily if one neglects the lattice motion and calculates $U(\mathbf{r})$ using a purely electrostatic model. This procedure has the advantage of allowing direct numerical calculation of $U(\mathbf{r})$ for any $\mathbf{r}$, thereby eliminating the need for relying on an extrapolation formula like Eq. (II.9a). Van Horn ${ }^{7}$ has shown that, in this static approximation,

and

$$
\Omega_{x}=1.85 \omega_{0},
$$

$$
\Omega_{z}=2.39 \omega_{0}
$$

for the bcc lattice structure.

\footnotetext{
$7 \mathrm{H}$. Yan Horn (private communication)
}

Comparison of Eqs. (II.18) with Eqs. (II.17) indicates that coupling to the lattice motion decreases the oscillator frequencies somewhat. The second derivatives $\partial^{2} V / \partial z^{2}\left(0,0, b_{\mathrm{nn}}\right)$ and $\partial^{2} V / \partial x^{2}\left(0,0, b_{\mathrm{nn}}\right)$ are reduced by $38 \%$ and $52 \%$, respectively, by the motion of the lattice. The lattice effectively polarizes under the influence of the motion of the two reacting particles. This polarization acts to reduce the Coulomb fields that oppose displacements of the reacting nuclei from their equilibrium positions. Lattice polarization increases the reaction rate noticeably. Figure 3 compares reaction rates computed using the static and dynamic values of $\Omega_{x}$ and $\Omega_{z}$.

\section{CALCULATION OF THE REACTION RATE}

In this section, we derive an expression for the reaction rate. We begin by finding a formula for the reaction rate in terms of the wave function corresponding to the non-nuclear potential

$$
V(\mathbf{r})=Z^{2} e^{2} r^{-1}+k_{2} r^{2}+k_{3} r^{3}+k^{\prime}\left(x^{2}+y^{2}\right) .
$$

In Secs. IIIB and IIIC, we derive the wave function, and in Sec. IIID we obtain the reaction rate itself.

\section{A. General Expression for the Reaction Rate}

The total potential affecting the relative motion of two reacting particles is the sum of the non-nuclear potential $V(\mathbf{r})$ of Eq. (III.1) and a nuclear potential. The nuclear potential is effectively zero except within a radius $R$, where

$$
R \ll b_{\mathrm{nn}},
$$

since we limit ourselves to densities well below nuclear densities.

We decompose the regular solution to the Schrödinger equation

$$
\left\{\nabla^{2}+2 \mu \hbar^{-2}[E-V(\mathbf{r})]\right\} \psi(\mathbf{r})=0
$$

in terms of spherical harmonics as follows:

$$
\psi(\mathbf{r})=\sum_{L M} a_{L M} f_{L}(E ; r) Y_{L M}(\Omega) .
$$

Let the regular solution to the Coulomb-wave Schrödinger equation

$$
\left\{\nabla^{2}+2 \mu \hbar^{-2}\left[E-Z^{2} e^{2} r^{-1}\right]\right\} \psi^{c}(\mathbf{r})=0
$$

be written

$$
\text { Since } \begin{gathered}
\psi^{c}(\mathbf{r})=\sum_{L M} a_{L M} f_{L}^{c}(E ; r) Y_{L M}(\Omega) . \\
V(\mathbf{r}) \approx Z^{2} e^{2} r^{-1}
\end{gathered}
$$

for $r \ll b_{\mathrm{nn}}$, the radial functions $f_{L}(E ; r)$ and $f_{L} c(E ; r)$ must differ only by a constant factor when $r$ is near the nuclear radius $R$, which is small compared to $b_{\text {nn }}$. Thus it is interesting to compare the reaction rate $\Gamma(E)$ for an external potential $V(\mathbf{r})$ with the rate $\Gamma^{c}(E)$ of the same reaction at the same energy but with an external potential $Z^{2} e^{2} r^{-1}$. 
We limit ourselves to reactions in which one incident orbital angular momentum value $L$ dominates the reaction rate. We also choose a $\psi^{c}(\mathbf{r})$ which approaches a plane wave of unit intensity as $r \rightarrow \infty$, except for the usual slowly varying phase factor characteristic of Coulomb waves. We normalize $f_{L}{ }^{c}(E ; r)$ such that

$$
f_{L}{ }^{c}(E ; r) \rightarrow(\kappa r)^{-1} \sin [\kappa r-\alpha(r)],
$$

as $r \rightarrow \infty$. Then one can show that the reaction rates for external potentials $V(\mathbf{r})$ and $Z^{2} e^{2} / r$ are related as follows:

$$
\frac{\Gamma_{L}}{\Gamma_{L} c}=\frac{\sum_{M}\left|a_{L M}\right|^{2}}{4 \pi(2 L+1)}\left|\lim _{r \rightarrow 0} \frac{f_{L}(E ; r)}{f_{L}^{c}(E ; r)}\right|^{2} .
$$

In the follow subsections, we find expressions for $a_{L M}$ and $f_{L}(E ; r)$ for substitution in Eq. (III.9).

\section{B. The Radial Equation}

The remaining problem is to solve Eq. (III.3) for $\psi(\mathbf{r})$. We concluded in Sec. II that the harmonic oscillator approximation is valid near the point $\left(0,0, b_{\mathrm{nn}}\right)$. Thus, near $\left(0,0, b_{\mathrm{nn}}\right)$ we can write

$$
\begin{aligned}
& \sum_{L M} a_{L M} f_{L}(r) Y_{L M}(\Omega) \\
& \quad=U_{x}\left(n_{x} ; x\right) U_{y}\left(n_{y} ; y\right) U_{z}\left(n_{z} ; z\right) .
\end{aligned}
$$

The right side of Eq. (III.10) represents a normalized three-dimensional harmonic oscillator wave function with frequencies $\Omega_{x}, \Omega_{x}$, and $\Omega_{z}$ and occupation numbers $n_{x}, n_{y}$, and $n_{z}$. The harmonic oscillator wave functions are large only near $x=0, y=0, z=b_{\mathrm{nn}}$, or, in other words, $r=b_{\mathrm{nn}}, \theta=0$. Thus, the product $U_{x}\left(n_{x} ; x\right) U_{y}\left(n_{y} ; y\right)$ essentially expresses the angular dependence of the wave function while $U_{z}\left(n_{z} ; z\right)$ describes the radial dependence. Hence, we can write

$$
f_{L}\left(n_{z} ; r\right) \approx U_{z}\left(n_{z} ; r\right) b_{\mathrm{nn}^{-1}}
$$

for $r$ near $b_{\mathrm{nn}}$ and

$$
\begin{aligned}
a_{L M}\left(n_{x} n_{y}\right) \approx b_{\mathrm{nn}}{ }^{-1} & \int_{-\infty}^{\infty} d x \int_{-\infty}^{\infty} d y U_{x}\left(n_{x} ; x\right) \\
& \times U_{y}\left(n_{y} ; y\right) Y_{L M} *[\Omega(x, y)] .
\end{aligned}
$$

In this approximation the coefficients $a_{L M}$ depend on $n_{x}$ and $n_{y}$, but not on $n_{z}$. We have shown that the radial wave function is independent of $n_{x}$ and $n_{y}$ for $r$ near $b_{\mathrm{nn}}$, and we shall show later that $f_{L}$ is approximately independent of $n_{x}$ and $n_{y}$ for smaller $r$.

We should note that the integration in Eq. (III.12a) can be performed readily for the important special case where $n_{x}=n_{y}=L=M=0$, and the result is

$$
a_{00}(0,0) \approx \hbar^{1 / 2}\left(\mu \Omega_{x} b_{\mathrm{nn}}^{2}\right)^{-1 / 2} .
$$

According to Eq. (III.11), $f_{L}\left(n_{z} ; r\right)$ must satisfy the same differential equation as $U_{z}\left(n_{z} ; r\right)$ for $r$ near $b_{\mathrm{nn}}$.
Thus, we find that

$$
\left[-\frac{d^{2}}{d r^{2}}+g_{1}(r)\right] f_{L}\left(n_{z}, r\right) \approx 0
$$

for $r$ near $b_{\mathrm{nn}}$. The quantity $g_{1}(r)$ is defined by

$$
\begin{array}{r}
g_{1}(r)=2 \mu \hbar^{-2}\left[V(0,0, r)-V\left(0,0, b_{\mathrm{nn}}\right)\right. \\
\left.-\left(n_{z}+\frac{1}{2}\right) \hbar \Omega_{z}\right] .
\end{array}
$$

We want to compare Eqs. (III.13) with the equation $f_{L}$ satisfies for small $r$. At small $r$, we can neglect the anisotropy of the potential and separate the solution into radial and angular components in the usual way. Then, for $r \ll b_{\mathrm{nn}}, f_{L}$ satisfies the equation

$$
\left[-\frac{d^{2}}{d r^{2}}+\frac{L(L+1)}{r^{2}}+g_{2}(r)\right]\left[r f_{L}\left(n_{z} ; r\right)\right]=0,
$$

where

$$
g_{2}(r)=g_{1}(r)-2 \mu \hbar^{-1} \Omega_{x}\left(n_{x}+n_{y}+1\right) .
$$

It would, of course, be convenient if $f_{L}\left(n_{z} ; r\right)$ satisfied the same differential equation for all $r$, $0<r<b_{\mathrm{nn}}$. We now show that the radial wave function approximately satisfies the differential equation

$$
\left[-\frac{d^{2}}{d r^{2}}+\frac{L(L+1)}{r^{2}}+g_{1}(r)\right]\left[r f_{L}\left(n_{z} ; r\right)\right]=0,
$$

both for $r \approx b_{\mathrm{nn}}$ and for $r \ll b_{\mathrm{nn}}$ by noticing that Eqs. (III.13a) and (III.15) are approximately the same for $r$ near $b_{\text {nn }}$ and that Eqs. (III.14a) and (III.15) are essentially equivalent for small $r$. Comparing Eqs. (III.13a) and (III.15) we note the following facts: (1) the term $L(L+1) r^{-2}$ in Eq. (III.15) is negligibly small for $r$ near $b_{\mathrm{nn}}$ providing the expectation value $\left\langle\left(z-b_{\mathrm{nn}}\right)^{2}\right\rangle$ is small compared to $b_{\mathrm{nn}}^{2}$; and (2) the quantity $r f_{L}\left(n_{z} ; r\right)$ can be accurately approximated by $b_{\mathrm{nn}} f_{L}\left(n_{z} ; r\right)$ for $r$ near $b_{\text {nn. }}$. It follows that Eqs. (III.13a) and (III.15) are essentially the same for $r$ near $b_{\mathrm{nn}}$. Comparing Eqs. (III.14a) and (III.15) for $r \ll b_{\text {nn }}$, we notice that the quantity $\epsilon_{x y}$ defined by

$$
\epsilon_{x y}=2 \mu \Omega_{x} \hbar^{-1}\left(n_{x}+n_{y}+1\right)
$$

is small compared to $2 \mu Z^{2} e^{2} \hbar^{-2} r^{-1}$. Thus Eqs. (III.14a) and (III.15) differ little for $r \ll b_{n n}$. We have now established that Eq. (III.15) holds accurately in the limits of large and small $r$. We assume that it holds approximately for intermediate $r$.

The most serious approximation involved in the use of Eq. (III.15) for all $r$ is the neglect of $\epsilon_{x y}$ for small and intermediate $r$. One can estimate the resultant error in the calculated reaction rate by adding $\epsilon_{x y}$ to the energy for small $r$ in the barrier penetration factor of Eq. (II.6). One finds that the error in the barrier penetration integral $I_{p}$ should be less than $2 \%$.

By making various approximations we have shown that the radial wave function satisfies Eq. (III.15) for 
all $r$. In Sec. IIIC we outline the procedure for integrating Eq. (III.15) to find $f_{L}\left(n_{z} ; r\right)$.

\section{The Radial Wave Function}

Our method of solving Eq. (III.15) approximately for $f_{L}\left(n_{z} ; r\right)$ is algebraically complicated but straightforward. It introduces errors small compared to those due to the approximations involved in Eq. (III.15) itself. Thus, we only outline the procedure briefly.

We use the modified WKB approximation ${ }^{8}$ in which the centrifugal potential is represented by $\left(L+\frac{1}{2}\right)^{2} r^{-2}$ instead of $L(L+1) r^{-2}$. We determine the normalization by matching the WKB approximation to the harmonic oscillator wave function for $r$ near $b_{\mathrm{nn}}$. The WKB integral cannot be evaluated analytically, but it can be expressed to a good approximation as the sum of two integrals which can be calculated exactly. The first integral is the one that appears in the WKB approximation to a Coulomb wave function. Thus, the radial wave function $f_{L}\left(n_{z} ; r\right)$ can be written as the product of a Coulomb wave function and a correction factor. The Coulomb wave function appearing in $f_{L}\left(n_{z} ; r\right)$ is $f_{L}{ }^{c}\left(E^{\prime} ; r\right)$, where

The relation

$$
E^{\prime}=Z^{2} e^{2} r_{c}^{-1}\left(1+\xi^{-1}\right) .
$$

$$
\xi=2 \mu Z^{2} e^{2} \hbar^{-2}\left(L+\frac{1}{2}\right)^{-2} r_{c}
$$

defines the parameter $\xi$, which is usually much larger than one. Thus, $E^{\prime}$ is approximately the energy of a pure Coulomb wave with classical turning point $r_{c}$. The classical turning point radius defined in Eq. (II.6c) can be expressed in the approximate form

$$
r_{c} \approx b_{\mathrm{nn}}-\left[\hbar\left(2 n_{z}+1\right)\right]^{1 / 2}\left(\mu \Omega_{z}\right)^{-1 / 2},
$$

providing the vibrations are small.

To find the reaction rate using Eq. (III.9), we must calculate the ratio $Q$ given by

$$
Q=\lim _{r \rightarrow 0}\left[f_{L}\left(n_{z} ; r\right) / f_{L}^{c}(E ; r)\right],
$$

where $E$ is defined in Eq. (II.8). The quotient $Q$ is the ratio of the Coulomb wave functions for energies $E^{\prime}$ and $E$ multiplied by a correction factor.

We must define four parameters occurring in the two Coulomb wave functions. The expressions

and

$$
\kappa=\hbar^{-1}(2 \mu E)^{1 / 2}
$$

$$
\kappa^{\prime}=\hbar^{-1}\left(2 \mu E^{\prime}\right)^{1 / 2}
$$

express the wave numbers in terms of the energies, while the equations

and

$$
\eta=Z^{2} e^{2} \mu \hbar^{-2} \kappa^{-1},
$$

$$
\eta^{\prime}=Z^{2} e^{2} \mu \hbar^{-2}\left(\kappa^{\prime}\right)^{-1}
$$

${ }^{8}$ F. L. Yost, J. A. Wheeler, and G. Breit, Phys. Rev. 49, 174 (1936). give the Coulomb field parameters in terms of the wave numbers.

We must also define some parameters occurring in the correction factor that multiplies the ratio of the Coulomb wave functions. Let and

$$
\zeta=2 \mu k_{2} \hbar^{-2}\left(L+\frac{1}{2}\right)^{-2} r_{c}^{4},
$$

$$
\sigma=2 \mu k_{3} \hbar^{-2}\left(L+\frac{1}{2}\right)^{-2} r_{c}^{5} .
$$

Then define $A, B, C$, and $D$ by the relations

$$
\begin{gathered}
A=(1 / 16)\left(3 \xi^{3}+4 \xi^{2}-4 \xi\right)(1+\xi)^{-7 / 2} \\
B=(1 / 128)\left(29 \xi^{4}+72 \xi^{3}+24 \xi^{2}-32 \xi-48\right) \\
\times(1+\xi)^{-9 / 2} \\
C=(1 / 24)\left(9 \xi^{2}+32 \xi+8\right)(1+\xi)^{-3}
\end{gathered}
$$

and

$D=(1 / 192)\left(87 \xi^{3}+356 \xi^{2}+356 \xi+192\right)(1+\xi)^{-4}$.

Finally, let

$$
I=\left(L+\frac{1}{2}\right)\left[\left(\cos ^{-1} \alpha\right)(\zeta A+\sigma B)+\zeta C+\sigma D\right],
$$

where

$$
\alpha=-\xi(\xi+2)^{-1} .
$$

Then one can show that

where

$$
Q=F \exp \left[\frac{1}{2} I-\pi\left(\eta^{\prime}-\eta\right)\right],
$$

$$
F=\left[\frac{2 \mu \Omega_{z}\left(\kappa^{\prime}\right)^{2 L}}{\pi h \kappa^{2 L-1}} \prod_{s=1}^{L}\left(\frac{1+\eta^{\prime 2} s^{-2}}{1+\eta^{2} s^{-2}}\right)\right]^{1 / 2} .
$$

The quantity $Q$ gives the ratio of the wave function $f_{L}\left(n_{z} ; r\right)$ to the Coulomb wave function for the energy $E$. We now use Eqs. (III.32) in Eq. (III.9) to find the reaction rate.

\section{The Reaction Rate}

We first consider the reaction rate from an initial state $\left(n_{x}, n_{y}, n_{z}\right)$. Substituting Eqs. (III.32) in Eq. (III.9) yields

where

$$
\Gamma_{L}\left(n_{x}, n_{y}, n_{z}\right)=G \Gamma_{L}^{c}(E),
$$

$$
\begin{aligned}
& G=[4 \pi(2 L+1)]^{-1} F^{2} \sum_{M}\left|a_{L M}\left(n_{x}, n_{y}\right)\right|^{2} \\
& \times \exp \left[I-2 \pi\left(\eta^{\prime}-\eta\right)\right],
\end{aligned}
$$

and $\Gamma_{L}{ }^{c}(E)$ is the reaction rate for a pure Coulomb wave with energy $E$.

To find the average lifetime of a nucleus in a stellar interior, we must perform a thermal average over oscillator states. We shall find in Sec. IV that the theory applies only to temperatures low enough that

$$
\hbar \Omega_{x}(k T)^{-1} \ll 1 .
$$

Thus, we assume $n_{x}$ and $n_{y}$ are both zero. The sum over $n_{z}$ must be carried out, however, due to the strong 
dependence of $\eta^{\prime}$ on $n_{z}$. Consistent with our previous assumption of a bcc lattice, we assume each nucleus has eight nearest neighbors and obtain the expression

$$
\tau_{L}^{-1}=8 \sum_{n_{z}=0}^{\infty} \Gamma_{L}\left(0,0, n_{z}\right) \exp \left[-n_{z} \hbar \Omega_{z}(k T)^{-1}\right]
$$

for the inverse lifetime.

In the important special case of an $s$-wave interaction, the reaction rate corresponding to a Coulomb wave with unit number density at infinity is often written ${ }^{9}$

$$
\Gamma_{0}{ }^{c}=S(E) v E^{-1} e^{-2 \pi \eta},
$$

where the cross-section factor $S(E)$ can usually be determined from the results of laboratory experiments; it contains all of the purely nuclear aspects of the reaction rate. The quantity $v$ in Eq. (III.36) is the velocity corresponding to energy $E$ and wave number к. Using Eqs. (II.17), (III.12b), (III.32b), (III.33), and (III.36) in Eq. (III.35), one finds that the inverse lifetime for an $s$-wave reaction is given by

$\tau_{0}^{-1}=J \sum_{n_{z}} S(E)$

$\times \exp \left[-2 \pi \eta^{\prime}+I-n_{z} \hbar \Omega_{z}(k T)^{-1}\right]$,

where

$$
J=1.00(\rho / M)^{2 / 3} \hbar^{-1} .
$$

The quantities $\eta^{\prime}$ and $I$ were defined in Eqs. (III.24) and (III.31), respectively. The energy $E$ can be written in the convenient form

$E=1.48 Z^{2} e^{2}(\rho / M)^{1 / 3}+1.88\left(n_{z}+\frac{1}{2}\right) \hbar Z e \rho^{1 / 2} M^{-1}$.

Equations (III.35) and (III.37) give the inverse lifetime of a nucleus in a solid lattice of density $\rho$. In Sec. IV we describe the range of temperatures and densities to which these formulas apply.

\section{LIMITATIONS AND GENERALIZATIONS}

\section{A. Assumption of Identical Particles}

We have considered so far only the case of nuclear reactions in a lattice of identical particles. The assumption of identical particles allowed the relatively easy evaluation of $b_{\mathrm{nn}}, \Omega_{x}$, and $\Omega_{z}$. If these parameters could be evaluated for a medium of more complicated composition, the rest of our treatment could immediately be generalized to include reactions between nonidentical particles. Equation (III.33) holds for nonidentical particles, providing we interpret $\mu$ as $M_{1} M_{2}\left(M_{1}+M_{2}\right)^{-1}$ and replace $Z^{2}$ by $Z_{1} Z_{2}$ in all cases.

Accurate evaluation of $b_{\mathrm{nn}}, \Omega_{x}$, and $\Omega_{z}$ is difficult for a nuclear reaction in a star of arbitrary composition. Such a star does not possess a periodic lattice. Consequently the phonon technique cannot be used to find $\Omega_{x}$ and $\Omega_{z}$, and typical distances between neighboring nuclei of the reacting species could only be estimated accurately by

${ }^{9}$ P. D. Parker, J. N. Bahcall, and W. A. Fowler, Astrophys. J. 139,602 (1964). careful analysis of the energies of different geometrical configurations.

Here we suggest a crude general rule for estimating the nearest-neighbor distance between two nonidentical nuclei. We picture the lattice as composed of neutral regions, one region for each ion. The neutral region including a nucleus of charge $Z^{\prime}$ would have volume $Z^{\prime} n_{e}^{-1}$, where $n_{e}$ is the electron number density. For example, consider the case of a nucleus of charge $Z_{1}$ imbedded in a medium of much smaller charges $Z_{2}$. We could picture the charge $Z_{1}$ at the center of a sphere of radius $\left(3 Z_{1}\right)^{1 / 3}\left(4 \pi n_{e}\right)^{-1 / 3}$. The sphere would then be surrounded by small cubes of edge length $Z_{2}{ }^{1 / 3} n_{e}{ }^{-1 / 3}$, each cube containing one nucleus and $Z_{2}$ electrons. According to this crude picture, the nearest-neighbor distance between nuclei of charge $Z_{1}$ and nuclei of charge $Z_{2}$ is given by

$$
b_{\mathrm{nn}}=n_{e}^{-1 / 3}\left[\left(3 Z_{1}\right)^{1 / 3}(4 \pi)^{-1 / 3}+\frac{1}{2} Z_{2}{ }^{1 / 3}\right] .
$$

Although Eq. (IV.1) was "derived" for the case of $Z_{1} \gg Z_{2}$, we note that it also gives a reasonable formula

$$
b_{\mathrm{nn}}=1.12 b
$$

for the case where $Z_{1}$ and $Z_{2}$ are equal. Thus Eq. (IV.1) would be a reasonable guess for all $Z_{1} \geq Z_{2}$.

We can make a correspondingly simple assumption about the lattice potential. We assume $U(\mathbf{r})$ has the form suggested in Eq. (II.9a), with $k_{3}$ set equal to zero. Then Eqs. (II.9b) and (II.9c) imply that

and

$$
\Omega_{z}{ }^{2}=3 Z_{1} Z_{2} e^{2} \mu^{-1} b_{\mathrm{nn}}{ }^{-3},
$$

$$
k_{2}=\frac{1}{2} Z_{1} Z_{2} e^{2} b_{\mathrm{nn}}{ }^{-3} .
$$

Setting $\Omega_{x}$ equal to $\Omega_{z}$ would not cause serious error since the reaction rate does not depend strongly on $\Omega_{x}$.

Equations (IV.1)-(IV.4) represent only crude estimates of the parameters needed for finding a reaction rate in a medium of arbitrary composition. Careful analysis of lattice configurations for various compositions might suggest more accurate rules.

\section{B. Assumption of One $L$ Value}

We have assumed that one initial value of orbital angular momentum dominates the reaction rate. Reactions between light nuclei are predominantly $s$ wave, but several different orbital angular momenta may be important in reactions between heavier nuclei. Incorrectly assuming that one $L$ value dominates the rate, one may overlook the effects of interference and may make errors in the geometrical factors $a_{L M}$, but such errors are unlikely to amount to as much as a factor of ten. The barrier penetration factors for reactions between heavy particles range from about $e^{-50}$ to $e^{-150}$ for the conditions to which the solid-state model applies. Owing to our incomplete knowledge of $U(\mathbf{r})$ and our approximate method of solving the Schrödinger 
equation, we are likely to make errors of several percent in the barrier penetration exponents. These errors are likely to be larger than any caused by incorrect assumptions about the dominant $L$ values.

\section{Resonant Reactions}

The treatment outlined above does not apply directly to reactions with strong resonances at energies smaller than about two or three times $Z^{2} e^{2} b^{-1}$, which ranges from less than $1 \mathrm{keV}$ for protons at $10^{5} \mathrm{~g} / \mathrm{cc}$ to several hundred $\mathrm{keV}$ for carbon nuclei at $10^{10} \mathrm{~g} / \mathrm{cc}$. The widths of the harmonic oscillator states are likely to be large compared to the widths of the nuclear resonances. To apply the solid-state treatment to a reaction like

$$
3 \mathrm{He}^{4} \rightarrow \mathrm{C}^{12}+\gamma,
$$

which involves low-lying resonances, one would have to estimate the widths of the oscillator states and replace the sum in Eq. (III.37a) by an integral.

\section{High-Density Limit}

At high densities, the amplitudes of the ground-state vibrations may become comparable to $b_{\mathrm{nn}}$. When this happens, the nuclei no longer form a bcc lattice, as assumed in Secs. II and III. Several investigators have estimated the "melting density" of a lattice consisting of electrons immersed in a uniform distribution of positive charge. These estimates can easily be converted to apply to the case of a lattice of nuclei in a uniform negative charge density. The most recent estimates are those by de Wette. ${ }^{10}$ His work locates the melting density in the range

$$
1.6 \times 10^{4} Z^{6} A^{4}<\rho_{m}<1.6 \times 10^{5} Z^{6} A^{4},
$$

where $\rho_{m}$ is in $\mathrm{g} / \mathrm{cc}$. Earlier work ${ }^{11}$ indicated a melting density of about $10^{6} Z^{6} A^{4} \mathrm{~g} / \mathrm{cc}$.

Just above the melting point, the nuclei form a fluid rather than a periodic lattice, but the motion is still largely vibrational. In this liquid range, where the mean free path between collisions is small compared to $b_{\mathrm{nn}}$ but the vibrations are still too large to allow a strictly periodic lattice, it still seems reasonable to treat the relative motion of two particles using the potential of Eq. (II.9). That potential depends on the assumption of a bcc lattice through the parameters $b_{\mathrm{nn}}, \Omega_{x}$, and $\Omega_{z}$. The nearest-neighbor distance varies only a few percent from one lattice structure to another. The frequencies $\Omega_{x}$ and $\Omega_{z}$ have been calculated for the fcc lattice and for a "smeared-out" lattice intended to resemble a liquid, and the values of $\Omega_{x}$ and $\Omega_{z}$ are within about $10 \%$ of the values obtained for the bcc lattice. Thus we conclude that the parameters $b_{\mathrm{nn}}, \Omega_{x}$, and $\Omega_{z}$ are nearly independent of the geometrical arrangement of the lattice,

${ }^{10}$ F. W. de Wette, Phys. Rev. 135, A287 (1964).

${ }_{11}$ P. Nozières and D. Pines, Phys. Rev. 111, 442 (1958) and N. F. Mott, Phil. Mag. 6, 287 (1961). although they depend strongly on the density and on the charge and mass of the nuclei. We therefore hope that the values of $b_{\mathrm{nn}}, \Omega_{x}$, and $\Omega_{z}$ for the bcc lattice also suffice for the range of densities where the nuclei execute small vibrations in a nonperiodic lattice. The range of applicability of the formula could then be extended to a density given by the approximate relation

$$
\rho_{c} \approx 10^{6} Z^{6} A^{4} \mathrm{~g} / \mathrm{cc} \text {. }
$$

The above considerations are important mainly for reactions between protons. At densities greater than about $10^{6} \mathrm{~g} / \mathrm{cc}$, a zero-temperature proton star could be described more accurately as a degenerate gas than as a solid. Thus the solid-state approach fails to apply to protons at densities well below those expected in neutron stars.

We have also assumed that the nearest-neighbor distance is large compared to the nuclear radius. Thus, the solid-state model applies only if

$$
\rho \ll 10^{14} \mathrm{~g} / \mathrm{cc} .
$$

\section{E. High-Temperature Limit}

The temperature enters the expression for the reaction rate through the sums over $n_{z}$ in Eqs. (III.33) and (III.37). Below a critical temperature $T_{c}$, given approximately by the relation

$$
T_{c} \approx 1200 Z A^{-1} \rho^{1 / 2},
$$

where $T_{c}$ is in ${ }^{\circ} \mathrm{K}$ and $\rho$ is in $\mathrm{g} / \mathrm{cc}$, essentially all reactions take place from the ground state. Thus, for $T \ll T_{c}$, the rate is independent of $T$. Near the temperature $T_{c}$, the first few excited states become important, and the rate begins to increase with temperature. At a temperature just slightly above $T_{c}$, most reactions take place from unbound states, and the solid-state approach fails. Just above the critical temperature, most of the nuclei in the lattice are still in their ground states because

and

$$
\hbar \Omega_{x}\left(k T_{c}\right)^{-1} \approx 2.4
$$

$$
\hbar \Omega_{z}\left(k T_{c}\right)^{-1} \approx 3.5 \text {. }
$$

However, the exceptionally energetic nuclei that are most likely to react have enough energy to break through the lattice. The mean free path between collisions of these unusually energetic nuclei is large compared to $b_{\mathrm{nn}}$, and they can be treated approximately as gas particles. Salpeter ${ }^{4}$ has developed a method for calculating reaction rates for $T \gg T_{c}$.

\section{NUMERICAL RESULTS}

\section{A. Proton-Proton Reactions}

Equations (III.37) have been used to calculate the mean lifetime of the protons in hydrogen stars at various temperatures and densities. The protons were assumed 
to undergo the reactions

and

$$
\mathrm{H}^{1}+\mathrm{H}^{1} \rightarrow \mathrm{H}^{2}+e^{+}+\nu
$$

$$
\mathrm{H}^{1}+e^{-}+\mathrm{H}^{1} \rightarrow \mathrm{H}^{2}+\nu .
$$

For densities greater than about $10^{5} \mathrm{~g} / \mathrm{cc}$, the extreme degeneracy of the electrons causes the capture reaction (V.2) to dominate the process of hydrogen burning.

Figure 2 , shows the temperature dependence of the mean lifetime at a density of $10^{5} \mathrm{~g} / \mathrm{cc}$. Below a critical temperature of about $2 \times 10^{5 \circ} \mathrm{K}$, the reaction rate is independent of temperature. Above about $10^{6} \mathrm{~K}$, the formula of Salpeter ${ }^{4}$ should be accurate.

\section{B. Carbon-Carbon Reactions}

The mean lifetimes of $\mathrm{C}^{12}$ nuclei in stars of pure carbon have also been computed. Two carbon nuclei may react to form the following products: $\mathrm{Mg}^{24}+\gamma$, $\mathrm{Na}^{23}+\mathrm{H}^{1}, \mathrm{Mg}^{23}+n, \mathrm{Ne}^{20}+\mathrm{He}^{4}$, and $\mathrm{O}^{16}+2 \mathrm{He}^{4}$. Equations (III.37) were used to calculate the mean lifetimes of the carbon nuclei, even though there is no reason to expect that the reactions are predominantly $s$ wave. Reeves $^{12}$ has expressed the rate of the carbon-carbon reactions in terms of the cross section parameter $S(E)$. The small errors caused by estimating the geometrical factors $a_{L M}$ incorrectly and by neglecting interference effects should not be serious because of the strong density dependence of the reaction rate.

Figure 3 shows the mean lifetime of a carbon nucleus at $10^{7} \mathrm{~K}$. At low temperatures the reaction rate is significant for densities greater than about $10^{10} \mathrm{~g} / \mathrm{cc}$. The rate of the $\mathrm{C}^{12}+\mathrm{C}^{12}$ reactions depends much more strongly on density than the rate of the proton-proton reactions because the barrier penetration exponent is much larger for $Z=6$ than for $Z=1$.

\section{Comparison with Cameron's Method}

Cameron $^{2}$ has suggested calculating the rates of pycnonuclear reactions by treating the system of nuclei as a gas with Coulomb interactions between the particles. The curves marked "GAS (CAMERON)" in Fig. 3 were computed by a method similar to that proposed by

FIG. 2. Predictions of proton lifetimes at $10^{5}$ $\mathrm{g} / \mathrm{cc}$. The lifetimes predicted by the method of Salpeter are compared to those computed by the solid-state method using oscillator frequencies obtained by analyzing the dynamics of the lattice. The dashed line indicates a reasonable interpolation between the two formulas.

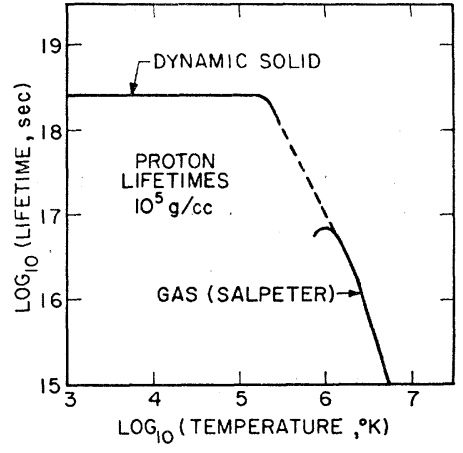

${ }^{12}$ H. Reeves, Astrophys. J. 135, 779 (1962).
Fig. 3. Predictions of the lifetimes of protons and $\mathrm{C}^{12}$ nuclei. The lifetimes predicted by the method of Cameron are compared to those computed by the solid-state method using oscillator frequencies obtained (1) from an analysis of lattice dynamics and (2) from an electrostatic analysis.
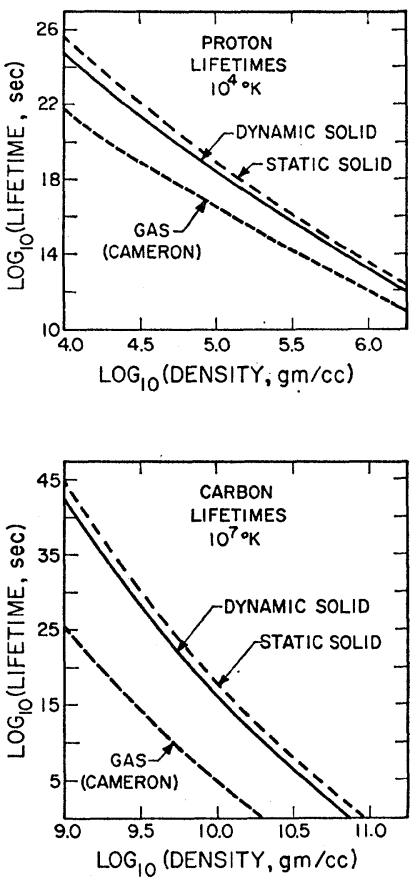

Cameron, using the same values of the cross-section parameter $S(E)^{9,12}$ as in the solid-state calculation.

Figure 3 indicates that the solid-state method predicts rates one to ten orders of magnitude smaller than those computed by the gas model. The large discrepancy in the predictions of the two models is due to the different estimates of the classical turning point radius $r_{c}$, which is an important factor in the barrier penetration exponent. According to the solid-state approach, $r_{c}$ is slightly less than the nearest-neighbor distance. According to Cameron's model of electrostatic screening at low temperatures, $r_{c}$ is slightly less than the charge-cloud radius, given by $\left(3 Z_{1}\right)^{1 / 3}\left(4 \pi n_{e}\right)^{-1 / 3}$, where $Z_{1} \geq Z_{2}$. For $Z_{1}=Z_{2}$, this charge-cloud radius is only 0.57 of our nearest-neighbor distance. Due to the strong dependence of the barrier penetration factor on the classical turning point, this factor of 0.57 causes a large difference in the predicted rates. However, for $Z_{1} \gg Z_{2}$, Cameron's charge-cloud radius is approximately equal to the nearest-neighbor distance given by Eq. (IV.1). Hence Cameron's method and the solid-state method would give similar predictions for reactions in which one nucleus is much larger than the other.

\section{ACKNOWLEDGMENTS}

I am grateful to Dr. J. N. Bahcall for his skillful guidance of this work and for his countless illuminating suggestions. Discussions with Professor R. F. Christy and Professor E. E. Salpeter have also been most helpful. I would like to thank H. Van Horn for informing me about his extensive work on the same problem and for calling my attention to several important papers. 